\title{
Fenomenología, sentimientos e identidad. La contribución de la fenomenología husserliana de los sentimientos a la cuestión de la identidad personal
}

\author{
Phenomenology, Feelings and Identity: \\ The Contribution of Husserlian Phenomenology \\ of Feelings to the Problem of Personal Identity
}

Mariano CRESPO

ICS/Universidad de Navarra

Recibido: 20/01/2015

Aceptado: 19/10/2015

\section{Resumen}

El artículo aborda la cuestión de la contribución de los sentimientos a la "conformación" y a la "revelación" de la identidad personal desde la perspectiva de la fenomenología husserliana. Para ello se parte de una breve descripción de qué entiende Edmund Husserl por "sentimientos" señalando el error de considerar esta esfera como una esfera homogénea. La distinción fundamental que cruza la misma es la existente entre sensaciones afectivas (no intencionales) y actos afectivos (intencionales). Manuscritos inéditos de Husserl a los que el autor ha tenido acceso amplían esta distinción y profundizan en los diversos tipos de intencionalidad afectiva. Una vez ofrecida una visión de conjunto sobre la concepción husserliana de los sentimientos, se analiza la contribución de éstos - entendidos en uno u otro sentido - a la conformación de los distintos, por así decir, "niveles" de identidad personal. De este modo, se reconstruye una suerte de "arqueología" de la identidad personal que va desde lo que Husserl denomina "fluyente presente viviente" hasta lo que en este trabajo se llama "identidad personal en sentido eminente".

Palabras clave: emociones, fenomenología, identidad, persona, sentimientos. 


\begin{abstract}
This paper deals, from the point of view of Husserlian Phenomenology, with the topic of the contribution of feelings to build and to reveal personal identity. The starting point is a brief description of what Husserl understood by "feelings". The founder of the phenomenological movement considered that it was wrong to think that the sphere of feelings or affective experiences was homogenous. On the contrary, it is crossed by an important distinction namely the distinction between affective sensations (non intentional) and affective acts (intentional). Some unpublished Husserl's manuscripts develop this analysis and go deep in the different kinds of affective intentionality. Once one I have presented a general view of the sphere of feelings, I consider the contribution of these experiences to the "conformation" and "manifestation" of the different personal identity's levels. So one tries to offer a sort of "archeology" of personal identity starting with what Husserl called "lebendige Gegenwart" to what we call "personal identity in pregnant sense".

Keywords: emotions, feelings, identity, person, phenomenology.
\end{abstract}

Si la fenomenología de Husserl consiste en la descripción de lo que se me da en cuanto se me da o, en otra formulación de este autor, en la descripción de la cosa en el cómo de sus determinaciones, ¿en qué medida puede ésta contribuir a la cuestión acerca de la identidad personal? La dificultad de responder a esta pregunta puede verse aumentada por la insistencia de Husserl en que ese "me" contenido en la expresión "lo que se me da" no es sino la conciencia transcendental. ¿No es, por el contrario, la identidad personal algo esencialmente vinculado con la facticidad del ser humano y, por consiguiente, fuera del interés de la fenomenología transcendental? Sin embargo, ésta no renuncia a ser una metafísica de la subjetividad concreta que se ocupa también de los problemas de la facticidad contingente entre los cuales se encuentra, ciertamente, el de la identidad personal ${ }^{1}$.

\footnotetext{
${ }^{1}$ Cf. E. Husserl, Cartesianische Meditationen und Pariser Vorträge, Husserliana I, Der Haag, Nijhoff 1973, 182. En su obra póstuma Welt und Unendlichkeit. Zum Problem phänomenologischer Metaphysik, Karl Alber, Freiburg/München 2014, L. Tengelyi examina la idea de Husserl de una metafísica fenomenológica. Ésta tendría un carácter "no tradicional" dado que no investigaría los principios y causas del ente en cuanto ente, sino ciertos "hechos originarios" o "proto-hechos" (Urtatsachen). Se trata del Ich als Urfaktum, die Welthabe des jeweiligen Ich, "die Innerlichkeit des Füereinanderseins als eines intentionalen Ineinanderseins y el Faktum einer Geschichtsteleologie" (L. Tengelyi, op. cit., 184-185) Una metafísica así entendida no sería, como la Fenomenología trascendental, una ciencia $a$ priori, sino una ciencia de lo fáctico. Estos hechos originarios se caracterizan por una cierta necesidad. Ello explica que, según Tengelyi, del análisis de estos hechos originarios surja una metafísica de la
} 
Por otra parte, en un artículo reciente Hanne Jacobs responde a la pregunta por la contribución de la fenomenología de Husserl a la discusión acerca de la identidad personal señalando que este método filosófico puede llevar a cabo tal contribución en la medida que las descripciones de la conciencia, en cuanto "dativo de la manifestación", pueden ofrecernos un "conocimiento adicional acerca de lo que significa experimentarse a uno mismo a través del tiempo, experimentarse a uno mismo como persona y experimentarse a uno mismo como la misma persona que fue antes" ${ }^{\text {"2 }}$. De este modo, se apunta a dos dimensiones centrales de la identidad personal, a saber, la permanencia del sí mismo a través del tiempo y la experiencia del sí mismo como persona (y como la misma persona a través del tiempo).

Relacionado con esto cabe hacerse la siguiente pregunta: ¿en qué medida lo que habitualmente denominamos emociones, afectos o sentimientos pueden contribuir al esclarecimiento de estas dos dimensiones centrales de la persona? Formulado en términos más generales, ¿hasta qué punto las vivencias afectivas o sentimientos contribuyen, por un lado, a la "construcción" de la identidad personal y, por otro lado, a la "manifestación" de la misma? Responder con precisión a esta cuestión filosófica central constituye más bien todo un programa de investigación, el cual, como es fácilmente comprensible, rebasa los límites de un trabajo como éste. Aquí se pretende tan sólo ofrecer algunas orientaciones para responder a esta pregunta dentro del contexto de la fenomenología husserliana.

En este orden de cosas, antes de ofrecer estas orientaciones es preciso esclarecer, aunque sea mínimamente, qué es lo que Husserl entiende por "sentimientos". En este punto y como es conocido, Husserl considera que se cometería un error importante si se considerara que esta esfera es una esfera homogénea. En uno de los primeros textos en los que el iniciador de la fenomenología aborda la cuestión de los sentimientos, a saber, el $\S 15$ de la quinta de las Investigaciones lógicas se plantea la diferencia fundamental que cruza esta esfera ${ }^{3}$. Me refiero a la existente entre sensaciones afectivas (Gefühlsempfindungen) y actos afectivos (Gefühlakte). En este punto y como él mismo reconoce, Husserl es deudor, aunque crítico, de la distinción que lleva a cabo Franz Brentano entre las sensaciones afectivas de dolor y de placer

\footnotetext{
"facticidad accidental" (zufällige Faktizität).

2 "If Husserl's phenomenology can contribute something to the discussion concerning personal identity, it is exactly because his phenomenological descriptions of consciousness can give us additional insight into what it means to experience oneself as continuous through time, to experience oneself as a person, and to experience oneself as the same person that one was before". (H. Jacobs, "Towards a Phenomenological Account of Personal Identity", en C. Ierna et al. (eds.), Philosophy, Phenomenology, Sciences, Phaenomenologica 200, Springer, New York 2010, 334

${ }^{3}$ E. Husserl, Logische Untersuchungen, Zweiter Band, Erster Teil, Untersuchungen zur Phänomenologie und Theorie der Erkenntnis, Hrsg. von U. Panzer, Husserliana (Hua) XIX/2, Martinus Nijhoff, Der Haag 1984, 401-409. (Investigaciones Lógicas, trad. de M. García Morente y J. Gaos, Editorial Revista de Occidente, Madrid 1976, 508ss.
} 
y el dolor y el placer en el sentido de actos afectivos [Gefühlakte] $]^{4}$. Las primeras tienen el carácter de estados de la conciencia, son fenómenos físicos, mientras que los segundos son actos intencionales, esto es, fenómenos psíquicos que, al igual que las representaciones y los juicios, se caracterizan por poseer una referencia intencional a un objeto. Dicho más sucintamente, ciertos sentimientos son vivencias intencionales y otros no. En este sentido, la diferencia descriptiva entre ambos tipos de "sentimientos" es tan clara que a Husserl le parece difícil poder pensar seriamente en sostener la unidad de un auténtico género entre estas clases de sentimientos.

En un manuscrito de investigación de principios de Diciembre de 1911, dedicado al análisis de los diversos tipos de Gemütsintentionalität, Husserl amplia estas consideraciones iniciales, realizadas diez años antes en Investigaciones lógicas, distinguiendo entre actos afectivos, reacciones afectivas y la unidad de disposición de ánimo (Stimmung) en cuanto "unidad de la coloración afectiva [Gefühlsfärbung] que la totalidad del continuo de la conciencia, la esfera toda de lo que aparece, recibe por transferencia, la corriente general del sentimiento en la que nadamos" ${ }^{5}$. En este manuscrito, Husserl llega incluso a adscribir un carácter intencional a este último tipo de vivencia afectiva:

"La intencionalidad del estado de ánimo, de la resolución firme como hábito, en la cual voy por la vida mientras que pienso, siento, hago esto o aquello, tiene siempre en el trasfondo mi fin, mi firme resolución, o la intencionalidad de la alegre y confiada disposición de ánimo o de la permanente certeza"

No es este el lugar de profundizar en los diversos tipos de vivencias afectivas o sentimientos, en la intencionalidad que les es propia, en sus semejanzas y diferencias con la intencionalidad teorética, en el interesantísimo fenómeno de la "coloración

\footnotetext{
${ }^{4}$ Cf. F. Brentano, Psychologie vom empirischen Standpunkt, Verlag Duncker and Humbold, Leipzig 1874 (reimpr. en Meiner Verlag, Hamburg 1973), § 3; cf. también I. Vendrell Ferrán, "La ética de las emociones de Francisco Brentano", en Anuario Filosófico, Vol. 45, Núm. 1, Abril 2012, 145-173.

5 "Also jedenfalls sind zu scheiden: die Gefühlsakte, die einzelnen Gefühlsreaktionen und die Einheit der Stimmung als die Einheit der Gefühlsfärbung, die der gesamte Bewusstseinsbestand, die gesamte Sphäre des Erscheinenden als solchen, durch Übertragung erhält, der allgemeine Strom des Gefühls, in dem wir schwimmen“ (Ms. A VI 12 II/73 a „H 21“, pp. 104-105). Este manuscrito pertenece a los Studien zur Struktur des Bewusstseins, una serie de manuscritos de investigación de Husserl cuya preparación para su publicación fue confiada por éste a Landgrebe y que en la actualidad están en fase de edición a cargo de Ulrich Melle y Thomas Vongehr, de los Archivos Husserl en Lovaina y que próximamente aparecerán en Husserliana. Sobre estos manuscritos cf. U. Melle, „Studien zur Struktur des Bewusstseins": Husserls Beitrag zu einer phänomenologischen Psychologie", en M. Ubiali, M. Wehrle (eds.), Feeling and Value, Willing and Action, Phaenomenologica 216, Springer, Dordrecht 2015, 3-11 y T. Vongehr, "Husserl über Gemüt und Gefühl in den Studien zur Struktur des Bewusstseins", en B. Centi y G. Gigliotti, (comps.), Fenomenologia della ragion pratica. L'etica di Edmund Husserl. Quaderni di Filosofia, 2, Bibliopolis, Napoles, 227-251

${ }^{6}$ E. Husserl, loc. cit.
} 
afectiva", etc. El propósito de esta sección inicial era ofrecer una visión panorámica de qué se entiende por "sentimientos" en el contexto de la fenomenología husserliana a fin de abordar la pregunta central que nos ocupa en este trabajo, a saber, cuál es el papel que los sentimientos tienen en la conformación y en la revelación de la identidad personal desde una perspectiva fenomenológica.

\section{II}

Como decía más arriba, la pregunta por el papel de los sentimientos - ya sean los estados psíquicos o las respuestas afectivas - en la conformación de la identidad personal tiene, si se me permite la expresión, como reverso la pregunta acerca de hasta qué punto este tipo de vivencias revelan, del modo que sea, la identidad personal de su sujeto. Dicho de otro modo, hasta qué punto lo que yo siento (tanto mis estados psíquicos como mis respuestas "sentimentales") y cómo lo siento revela quién soy.

Relacionado con esto, y como Max Scheler con su teoría de la estratificación de la vida emocional ${ }^{7}$, Alexander Pfänder ${ }^{8}$ con su concepción del yo y más recientemente Roberta de Monticelli ${ }^{9}$ recuerdan, en el lenguaje común se considera que hay experiencias afectivas que revelan más que otras la profundidad o interioridad de la persona, aquello que ellas son. Sentimientos como la tristeza, la alegría, la melancolía, el sentirse afortunado o desafortunado revelan no sólo una esfera emocional y una esfera axiológica "más profunda" que las reveladas por sentimientos como el dolor y el agrado sensibles, el placer o el gozo, sino que, al mismo tiempo, manifiestan, si cabe hablar así, "niveles" de identidad personal o planos antropológicos diferentes ${ }^{10}$.

\footnotetext{
${ }^{7}$ M. Scheler, Der Formalismus in der Ethik und die materiale Wertethik: neuer Versuch der Grundlegung eines ethischen Personalismus. Herausgegeben von Christian Bermes unter Mitarbeit von Annika Hand, Meiner, Hamburg 2014 (Ética, Introducción y edición de Juan Miguel Palacios, Madrid: Caparrós, 2001, 444-463). Scheler se refiere a esta teoría de la estratificación de la vida emocional, asimismo, en "El sentido del sufrimiento", en Amor y conocimiento y otros escritos. Edición y traducción de Sergio Sánchez-Migallón, Biblioteca Palabra nº 38, Ediciones Palabra, Madrid 2010, 49-114

8 Cf. A. Pfänder, Zur Psychologie der Gesinnungen I, en Jahrbuch für Philosophie und phänomenologische Forschung. 1. Band. In Gemeinschaft mit Oskar Becker (Freiburg), Moritz Geiger (München), Martin Heidegger (Freiburg), Alexander Pfänder (München), Adolf Reinach (Göttingen), Max Scheler (Berlin), herausgegeben von E. Husserl, Max Niemeyer Verlag. Halle a. S. 1913; A. Pfänder, Zur Psychologie der Gesinnungen, II en Jahrbuch für Philosophie und phänomenologische Forschung. 3. Band, Halle 2016. Cf. también U. Ferrer, Desarrollos de ética fenomenológica, Segunda edición, Editorial Moralea, Albacete 2002; M. Crespo, "Un capítulo de la fenomenología de la razón emotiva. El análisis de las disposiciones de ánimo según Alexander Pfänder”, en Pensamiento, Vol. 56, 2009, 413-431. ,d. cit.,eben damit in respecto. Este es uno de ellos:

${ }^{9}$ R. De Monticelli, L'ordine del cuore Etica e teoria del sentire, Garzanti, Milano 2012.

${ }^{10}$ Esta es la tesis central de S. Sánchez-Migallón, "La estratificación de la vida emocional según Scheler", en García de Leániz, I. (ed.), De nobis ipsis silemus. Homenaje a Juan Miguel Palacios, Ediciones Encuentro, Madrid 2010,357-376 y de De Monticelli, op. cit.
} 
En cualquier caso, lo anterior revela la necesidad de una "arqueología" de la identidad personal en el curso del devenir que parte del fluir anónimo originario hasta alcanzar la identidad personal en sentido propio e incluso lo que aquí llamaré "identidad personal en sentido eminente" 11 . Nos encontramos, pues, con dos extremos en este devenir: por un lado, lo que Husserl denomina "fluyente presente viviente" y, por otro lado, ese mismo yo concreto en cuanto persona única e irrepetible ${ }^{12}$.

Este es, pues, el contexto en el que surge la identidad personal en la perspectiva de la fenomenología husserliana. No es mi intención llevar a cabo en este trabajo una reconstrucción sistemática de ésta. Tan sólo quisiera referirme a algunos de los momentos más significativos de ella y al papel que los sentimientos - ya sean los estados psíquicos o las respuestas afectivas - desempeñan en éstos ${ }^{13}$.

Uno de los momentos iniciales de esta arqueología de la identidad personal es el constituido por lo que Husserl denomina "yo puro", a saber, el yo-polo que acompaña a todas mis vivencias como "sujeto" o "centro funcional" (Funktionszentrum) $)^{14}$ de las mismas.

"Observando, percibo yo algo; de igual modo, con mucha frecuencia estoy yo en el recuerdo "ocupado" con algo; cuasiobservando sigo yo en la fantasía fingidora al trajinar que tiene lugar en el mundo fantaseado. O bien, yo medito, yo saco conclusiones; yo me retracto de un juicio, eventualmente 'absteniéndome' sin más de juzgar. Yo llevo a cabo un agradarme o desagradarme, yo me alegro o estoy triste, yo deseo, o yo quiero y hago; o, también, yo 'contengo' la alegría, el deseo, el querer y el obrar. En todos estos actos yo participo, participo ACTUALMENTE. Al reflexionar, me aprehendo participando como este hombre" 15

\footnotetext{
${ }^{11}$ Cf. J.V. Iribarne, De la ética a la metafisica. En la perspectiva del pensamiento de Edmund Husserl, San Pablo, Bogotá 2007, 83.

${ }^{12}$ Cf. K. Held, Lebendige Gegenwart: die Frage nach der Seinsweise des transzendentalen Ich bei Edmund Husserl, entwickelt am Leitfaden der Zeitproblematik, Martinus Nijhoff. Der Haag 1966. En De la ética a la metafísica Iribarne se refiere a la interesantísima cuestión de la temporalidad de este fluyente presente viviente que es el flujo originario de la conciencia.

${ }^{13}$ Julia Iribarne habla de "eslabones" de este proceso de surgimiento de la identidad personal. Quizá este término no sea el más feliz dado que puede suscitar la impresión de que se trata de un proceso discreto cuando en realidad, si se me permite la simplificación, se trata más bien de un continuo. El polo extremo de la identidad personal incorpora, lógicamente, los momentos anteriores del proceso "arqueológico" que conduce a él.

${ }^{14}$ E. Husserl, Zur Phänomenologie der Intersubjektivität. Zweiter Teil, Hrsg. v. I. Kern, Husserliana XIV, Martinus Nijhoff, Der Haag 1973, 30

${ }^{15}$ E. Husserl, Ideen zu einer reinen Phänomenologie und phänomenologischen Philosophie. Erstes Buch: Allgemeine Einführung in die reine Phänomenologie. Neu herausgegeben von Karl Schuhmann. Husserliana III/1, Martinus Nijhoff, Der Haag 1976, 268 (Ideas relativas a una fenomenología pura y una filosofía fenomenológica. Libro primero. Introducción general a la fenomenología pura. Nueva edición y refundición integral de la traducción de José Gaos por Antonio Zirión Quijano, Unam Instituto de Investigaciones filosóficas, Fondo de Cultura Económica, México D.F 2013, 179).
} 
Ahora bien, este yo que - en cuanto sujeto - acompaña a todas mis vivencias no puede él mismo ser hecho objeto de una vivencia o ser considerado como un momento efectivo de la vivencia misma al modo de un fragmento integrante ${ }^{16}$. Se trata, pues, del sujeto idéntico de todos los actos de la misma corriente de conciencia. Es, como dice Husserl, el centro de irradiación (Ausstrahlungszentrum) $)^{17}$ "de todo atender, captar, referir, vincular, de todo tomar posición teórico, valorativo, de todo estar alegre y estar triste, esperar y temer, hacer y padecer, etc." 18 , el yo que "evidentemente permanece ahí indiviso y numéricamente idéntico, mientras que vive en estos múltiples actos (...)"19.

La constatación de que yo soy el sujeto idéntico de todas las vivencias de mi conciencia - también de los estados psíquicos y de los actos del sentimiento - y de que, por consiguiente, existe una continuidad de mi yo a través del tiempo (y del sueño) constituye uno de los momentos iniciales de la arqueología de la identidad personal. Sin embargo, una teoría acerca de ésta debe "ofrecer algo más" que esta identidad meramente formal. ${ }^{20}$ Ser persona es mucho más que ser sujeto de una corriente de vivencias tanto teóricas como prácticas (estimativas y volitivas).

En este contexto, la identidad personal se abre, a mi juicio, en una cuádruple dirección: (1) la persona en cuanto conciencia encarnada, corpórea; (2) la persona en cuanto sustrato de habitualidades; (3) lo que me atrevería a llamar, la identidad

\footnotetext{
${ }^{16}$ E. Husserl, Ideen zu einer reinen Phänomenologie und phänomenologischen Philosophie. Erstes Buch: Zweites Buch: Phänomenologische Untersuchungen zur Konstitution, Husserliana IV, herausgegeben von Marly Biemel, Martinus Nijhoff, Der Haag 1952, 103 (Ideas relativas a una fenomenología pura y una filosofía fenomenológica. Libro segundo: Investigaciones fenomenológicas sobre la constitución. Traducción de Antonio Zirión, Universidad Nacional Autónoma de México, México D.F 1997, 139 ,d. cit.,eben damit in respecto. Este es uno de ellos:

17 "Ausstrahlunhgspunkt, Tätigkeitszentrum von Tätigkeiten, von Akten” (Hua XIV, 30)

${ }^{18}$ Hua IV, 105 (Ideas II, 141-142).

${ }^{19}$ Hua IV, p. 98 (Ideas II, 134). Es importante no perder de vista que el yo puro no es solamente un yo ejecutante en la actualidad de la conciencia, sino que también lo es en la inactualidad: "El yo no puede desaparecer jamás, siempre está en sus actos; pero ahora depende: si éstos son o se vuelven actos actuales, entonces el yo, por decirlo así, se presenta en ellos, sale a la luz, ejerce una función actual viva, se dirige en un rayo actual a lo objetivo; o si es por así decirlo un yo latente, entonces no lanza una mirada actual a algo: experimenta, obra, padece, no actualmente" (Hua IV, 100 / Ideas II, 136)). Resulta muy interesante la tesis de Jacobs sobre el mundo como garante de la identidad personal (cf. art. cit.) 20 "A theory of personal identity, however, most often aims to account for more than the continuity of consciousness through time and sleep. That is, a theory of personal identity aims to account for how I can still consider myself at point $Y$ to be the same person as at an earlier point X.". (Jacobs, 2010); "Even if we were to change our minds constantly, our personal history would, from a phenomenological perspective, still be characterized by some identity. That is, through all changes and self-transformations, it is always still me that is taking a stance in response to something that strikes me or exercises an appeal on me to take a stance. As the one feeling addressed to take a stance as well as being the agent and solely responsible one in taking such a stance, I do not endure like any worldly object, nor do I abide in the way that my habitualities do. As an agent considered apart from my initiatives, I am a mere formal identity, I." (Jacobs, art. cit., 350)
} 
personal en sentido propio y (4) la identidad personal en sentido eminente. Veamos en qué consiste cada una de estas dimensiones y cuál es la contribución de los sentimientos en cada una de ellas.

En primer lugar, se trata del hecho de ser conciencias encarnadas, corpóreas. Cada una de las conciencias configura el mundo desde su aquí respectivo. Como es sabido, los análisis de Husserl al respecto parten del carácter escorzado de la percepción espacial. Todo escorzo o apariencia en perspectiva implica no sólo algo que aparece, sino al mismo tiempo alguien ante quien aparece. Como alguien formuló felizmente, toda apariencia tiene su genitivo y su dativo. Este dativo es un cuerpo localizado en el espacio. De esta forma, el cuerpo es caracterizado como el punto cero (Nullpunkt), en relación con el cual el objeto se orienta ${ }^{21}$. Como Dan Zahavi ha señalado, estas reflexiones husserlianas acerca del cuerpo como condición de posibilidad de la intencionalidad perceptiva son radicalizadas en la medida en que Husserl llama la atención sobre el hecho de que movimientos como los de los ojos, de la mano, etc. presuponen un tipo particular de auto-sensibilidad corpórea ${ }^{22}$. Estas vivencias - que se ejecutan al mismo tiempo y que no son temáticas - de la posición y del movimiento de nuestro cuerpo son las experiencias cinestésicas, las cuales desempeñan una doble función: contribuyen a la aparición (constitución) de la cosa y, al mismo tiempo, a la aparición ante la conciencia (constitución) del cuerpo y, por tanto, de la identidad corpórea ${ }^{23}$. En la medida en que las cinestesias son sensaciones (Empfindungen), aunque no intencionales, podemos decir que nos encontramos ante una primera contribución de los sentimientos a la identidad personal.

Sin embargo, no hay que perder de vista que lo que hemos señalado tiene que ver con la constitución de la identidad personal encarnada en un cuerpo vivo vinculada al reconocimiento de este cuerpo como cuerpo propio. En lo que concierne a la exterioridad del cuerpo esta conciencia de identidad sólo se alcanza por la mediación del otro. En experiencia solipsista - utilizando la terminología

\footnotetext{
${ }^{21}$ Cf. Hua IV, 298 y E. Husserl, Phänomenologische Psychologie. Hrsg. v. W. Biemel, Husserliana IX, Martinus Nijhoff, Der Haag 1962, 392.

${ }^{22}$ D. Zahavi, Husserl's Phenomenology, Stanford University Press, Stanford 2003, 99.

23 "Wir ersehen (...), dass in der Tat die bloß visuellen Verläufe für die Auffassung nicht ausreichen, dass sie die Mittel nicht in sich haben, Ruhe und Bewegung zu unterschiedener Erscheinung zu bringen. Damit ist aber gesagt, dass die Konstitution der objektiven Lage und objektive Räumlichkeit wesentlich vermittelt ist durch die Leibesbewegung, phänomenologisch gesprochen, durch die kinästhetischen Empfindungen, sei es durch konstante, sei es durch verlaufende, durch kinästhetische Verläufe" (E. Husserl, Ding und Raum. Vorlesungen 1907. Hrsg. von Ulrich Claesges. Husserliana XVI, Martinus Nijhoff, Den Haag 1973, 176); „Also fungieren für die Dingerscheinung sowohl anderer Dinge wie des Leibes und andererseits als lokalisierte im Leib. Was liegt in dieser doppelten Auffassung? Jedenfalls: Mein Leib, im großen und ganzen in derselben Erscheinungsweise erscheinend, während die kinästhetischen Verhältnisse sich ändern, bewegt sich ,mit mir" ebenso, wie jedes andere Objekt, das bei kinästhetischen Veränderungen immer gleich erscheint, sich bewegt, und bewegt sich ,mit mir', da ich dabei eben mich auch bewege" (Hua XVI, 282)
} 
de Husserl - no alcanzamos el darnos a nosotros mismos como una cosa espacial como todas las demás.

Una segunda dirección de la identidad personal tiene que ver con la institución de habitualidades. Veamos en qué sentido. El yo no desempeña solamente una función activa en cuanto sujeto o centro de irradiación de las vivencias (Ausstrahlunhgspunkt), sino que también tiene una función, por así decir, pasiva en cuanto centro de recepción de irradiación (Einstrahlungspunkt), en cuanto sujeto de afecciones ${ }^{24}$. El yo puro no sólo va hacia el objeto sino que, al mismo tiempo, desde éste vienen hacia el yo "contrarrayos" (Gegenstrahlen). En el caso, por ejemplo, de determinadas decisiones importantes en mi vida no sólo soy el sujeto de las mismas, sino que éstas me "moldean" de alguna forma como la persona que soy.

La cuestión, cuyo debate aquí se inicia, es muy importante de cara a la identidad personal. De lo que aquí se trata es de que algunos objetos a los cuales se dirige el yo puro a través de determinadas vivencias intencionales dejan su "huella" en el propio yo. Esta "huella" tiene, en muchas ocasiones, un carácter "sentimental".

Esta capacidad del yo en virtud de la cual sus objetos pueden participar, de algún modo, en él pone de relieve que "este yo centrípeto no es un vacío polo de identidad (...) sino que, en virtud de una ley de la 'génesis trascendental', gana una nueva propiedad duradera con cada acto de un nuevo sentido objetivo irradiado por él" 25 . Dicho de otro modo, la "huella" que el objeto de la vivencia puede dejar en el yo contribuye a que éste se dirija (o, eventualmente, se abstenga de dirigirse) a los objetos de un modo determinado. De esta forma, el yo se constituye en un sustrato de habitualidades. "Me encuentro a mí mismo como el yo que es de esta opinión, que está determinado como un yo persistente por este hábito duradero"26.

Estas habitualidades permiten el surgimiento de "horizontes de capacidades", los cuales constituyen no una unidad estática, sino una "unidad en el estilo a través del cambio" 27 . Ahora bien, no todas las habitualidades son iguales, sino que existen

\footnotetext{
${ }^{24}$ Cf., por ejemplo, Hua XIV, 30ss.

${ }^{25}$ Hua I, 100

${ }^{26}$ Hua I, p. 101. "Das Ich vollzieht Aktivitäten. Aber es ist nicht ein leerer ideeller Polpunkt, bloß bestimmt als Schnittpunkt der aus ihm hervorströmenden und dann vorgegebenen Aktivitäten; sondern eben damit ineins Pol von entsprechenden Habitualitäten. Das sind aber nicht an ihm als wie $<$ an $>$ einem sachlichen Gegenstand wahrnehmungsmäßig gegebene und aufweisbare sachliche Eigenschaften, sondern Beschaffenheiten, die ihm aus der Genese, aus der Tatsache, daß es den jeweiligen actus vollzogen hat, zuwachsen und nur in Rekurs auf diesen historisch ihm zugehören. Mit der ursprünglichen Entscheidung wird das Ich ursprünglich zu dem so entschiedenen. Es kann sich nun alsbald als das erschauen und sich später als dasselbe erschauen, als dasselbe noch immer so entschiedene.“ (Hua IX, 211)

${ }^{27}$ Iribarne, op. cit., 95; Hua IX, 215; “Die Gesetzmäßigkeit der transzendentalen Genesis der Identität des reinen Ich hat zur Folge, dass das Ich durch die sich immer wandelnden und es motivierenden Zustände einen bleibenden Stil erhält, weil die Erwerbung jeder Habitualität ihm einen Horizont eröffnet, der zunächst ein neuer Horizont seiner selbst ist” (M. Ubiali, „Husserls Phänomenologie des
} 
diferentes estratos de las mismas que van desde aquellas que tienen su sede en el cuerpo propio animado (habitualidades relativas a patrones de percepción, de comportamiento, etc.) a habitualidades, por así decir, sancionadas por nuestra libertad $\mathrm{y}$, que, por consiguiente, tienen relevancia moral ${ }^{28}$. El primer tipo de habitualidades corresponden a lo que Husserl denomina seelische Ich o, simplemente, Seele (alma) entendido como un "complejo de disposiciones naturales que, como tales, están condicionadas por la naturaleza física y son dependientes de ellas" 29 . El segundo tipo de habitualidades apunta al ámbito de lo que el fundador del método fenomenológico denomina Geist (espiritu), el cual se edifica sobre el subsuelo del alma, tal y como Husserl entiende a ésta ${ }^{30}$.

Pues bien, es justamente sobre esta dimensión o "vida" espiritual en donde aparece la tercera dimensión de identidad personal, aquella que he denominado "identidad personal en sentido propio". A esta dimensión pertenecen los actos de la razón teórica, pero también los de la razón estimativa y los de la volitiva. Se trata, en términos de Husserl, del "yo específicamente espiritual, el sujeto de los actos del espíritu, la personalidad"31. "El espíritu no es un yo abstracto de los actos que toman posición, sino que es la personalidad plena, yo-hombre, el yo tomo posición, el yo pienso, valoro, actúo, llevo a cabo obras, etc." ${ }^{\prime 2}$ La persona, en cuanto sujeto que piensa, siente, valora, aspira, actúa, es atraído, repelido, se aficiona, se fastidia, desea y quiere, está en cada uno de estos actos en referencia a objetos de su mundo circundante (Umwelt). En estos actos el yo es "activo"; es un "yo que toma posición" (stellungnehmende Ich). Pero, como Husserl señala, este es sólo uno de los lados. Frente al activo está el yo pasivo, en el sentido de afectivo como de receptivo.

Ahora bien, como Jacobs señala, nuestra identidad personal no surge simplemente experimentando pasiva y receptivamente el mundo, sino que nos hacemos las personas que somos en la medida en que llevamos a cabo un tipo específico de acto intencional, a saber, las mencionadas "tomas de posición" (Stellungnahmen) ${ }^{33}$.

\footnotetext{
Habitus und der Konstitution des bleibenden Charakters", en M. Ubiali, M. Wehrle (eds.), Feeling and Value, Willing and Action, ed. cit., 116)

${ }^{28}$ Cf. Iribarne, op. cit., 102

${ }^{29}$ Hua IV, 281/Ideas II, 330

${ }^{30}$ Hua IV, 275/Ideas II, 323. Es importante no perder de vista que el Geist no es una anexo del cuerpo, sino que ambos constituyen una komprehensive Einheit (Cf. Hua IV, 236/Ideas II, 283.

${ }^{31}$ Husserliana IV, p. 276/Ideas II, 324. Husserl insiste en la dependencia de este nivel del nivel "natural": Este yo específicamente espiritual, el sujeto de los actos del espíritu, la personalidad, se halla a si dependiente de un subsuelo oscuro de predisposiciones de carácter, disposiciones primigenias y latentes, y por otro lado dependiente de la naturaleza" (ibid)

${ }^{32}$ Hua IV, 327

${ }_{33}^{33}$ Jacobs, art. cit., 345. Jacobs cita algunos pasajes de Husserl al respecto. Este es uno de ellos: "Ich kann Person nur sein, sofern ich nicht nur bleibende Apperzeptionen habe und durch sie eine standhaltende und mir als ich fremd gegenüberstehende Welt, sondern sofern ich bleibende „Überzeugungen“ habe, selbsterworbene, selbsttätig gewonnene Überzeugungen, durch tätige Stellungnahmen vom Ich her,
} 
Como acertadamente señala esta autora, localizar el ser persona en estas tomas de posición implica que ser esta persona y no otra no consiste tanto en tener una serie de rasgos, sino en que mis tomas de posición y las habitualidades que surgen de éstas se originan en mí individualmente. "Mis tomas de posición me hacen la persona que soy no por su contenido, sino por el hecho de que yo he optado por ellas" ${ }^{\prime 34}$.

Como se desprende de los ejemplos de tomas de posición que he dado, éstas no se limitan al ámbito teórico, sino que se dan también en las esferas evaluativa o estimativa y en la volitiva. Dado que los actos que pertenecen a estas dos últimas esferas son, según Husserl, Gefühlakte, podemos decir que los Gefühle (sentimientos intencionales) desempeñan un papel importante en la constitución de la identidad personal. Permítaseme un par de palabras al respecto.

Los objetos del mundo circundante, frente a los cuales el yo se muestra activo en sus tomas de posición, son constituidos en actos de este yo. Algunos de estos objetos se le dan al yo como bienes, objetos de uso, etc. Éstos remiten a actos valorativos, esto es, Gefühlakte, en los cuales las "meras cosas" recibieron este, en palabras de Husserl, "nuevo estrato de ser". ¿En qué consiste esta referencia intencional que el yo establece en los Gefühlakte con los objetos intencionales de éstos? Husserl inicia la respuesta a esta importante pregunta poniendo de manifiesto que esta referencia "no es inmediatamente una referencia real, sino una referencia intencional a algo real." ${ }^{35}$. Ambos tipos de referencia son radicalmente diferentes. Así, por ejemplo, la referencia real se suprime cuando la cosa no existe mientras que la referencia intencional permanece. Lo que aquí nos importa destacar es que la referencia de

bleibende Wertungen, bleibenden Willen" (Hua XIV, 196).

34 "As long as I am the author of my position-takings, however, they are mine and not yours, although we might share a set of decisions and convictions. Thus, it is not by their content that my convictions individualize me; rather, they receive their individualizing force from the fact that I have opted in favor of them" (Jacobs, art. cit., 347) Jacobs señala también cómo este planteamiento lleva a Husserl a sostener no sólo una "genuina teoría de la identidad personal", sino una teoría del "auténtico, verdadero sí mismo", el cual se expresa en la llamada ejercida por ciertos valores que, a pesar de ser personales, no dejan de ser objetivos. Para apoyar esta idea cita dos fragmentos de manuscritos husserlianos: "Das Gut, dem man entsagt, das man opfert, ist und bleibt ein Liebeswert, ein Wert für mich und als personale Individualität bin ich in solcher Wahl mit mir selbst in Widerstreit; indem ich das eine Gut opfere, opfere ich mich selbst; und der Schmerz des Opfers ist unüberwindlich" (Ms. A V 21/81b). Or, again, in another unpublished manuscript: "Bei Werten, die aus den Tiefen der Persönlichkeit und ihrer persönlichen Liebe ihren persönlichen Sinn empfangen, gibt es keine Wahl und keine „quantitativen“ Unterschiede, nämlich keine Unterschiede des Gewichtes, des überwiegenden und überwogenen. Ein Wert, der aus mir selbst entquillt, für den ich mich, als der ich bin, entscheide aus ursprünglich liebender Hingabe, ist praktisch ein unbedingter, ein absolut gesollter, mich bindend als der ich bin. Gegen ihn entscheiden ist sich selbst untreu werden, sich selbst verlieren, sich versündigen, sein wahres Ich verraten, seinem wahren Sein zuwider handeln (absoluter praktischer Widerspruch)" (Ms. B I 21/53a). Sobre este punto cf. también M. Crespo, "El amor como motivo ético en la fenomenología de Edmund Husserl”, en Anuario Filosófico, 45/1, 5-32.

${ }^{35}$ Hua IV, 215/Ideas II, 262 
un Gefühlakt como, por ejemplo, un acto valorativo (o cualquier otro tipo de toma de posición ya sea teórica o afectiva) es una referencia peculiar al objeto valorado, digamos, como "bueno". No es una referencia de la causalidad real, de la "causación en el sentido de la naturaleza", sino de la "causalidad subjetivo-objetiva". Para denominar este tipo de referencia Husserl utiliza las expresiones "causalidad de la motivación" o, simplemente, "motivación".

Es justamente al sujeto de la intencionalidad motivadora el que, según Husserl, hay que comprender como el yo espiritual o personal. De este modo, la motivación se convierte en la ley de la vida espiritual o personal ${ }^{36}$ de forma que en el "yo puedo" que instauran las habitualidades haya que distinguir entre el "yo puedo" físico, corporalmente mediado, y el espiritual o persona ${ }^{37}$.

La diferencia establecida entre el yo físico o corporal y el yo espiritual personal así como entre los dos tipos de causalidades que obran en ellos no debe llevar a pensar que se trata de dos yoes diferentes. Lo que realmente sucede es que el segundo se constituye sobre o fundado en el primero. Ciertamente, el yo personal se constituye sobre la primordialidad de los "instintos primigenios". Sin embargo, se trata de un "yo superior, autónomo, libremente actuante, guiado en particular por motivos de razón, no meramente arrastrado y no libre"38. Aunque Husserl no lo afirme directamente, es claro que los Gefühlakte, en sus variedades, desempeñan un papel muy importante en este proceso de conformación del yo personal.

Por último, una cuarta dirección o dimensión de la identidad personal es lo que llamábamos "identidad personal en sentido eminente". Aquí se apunta a la índole peculiar (Eigenart) de cada persona, la cual se expresa no sólo en el cómo llevo a cabo determinadas acciones físicas, sino sobre todo en el cómo de

"mis valoraciones individuales, mis maneras de dar preferencia, mis tentaciones, mis fuerzas para sobreponerme frente a ciertos grupos de tentaciones contra las que soy inmune; otro es en esto distinto, tiene otros motivos favoritos, otras tentaciones peligrosas para él, otras esferas de fuerza de acción individual, etc., pero dentro de la normalidad, específicamente la normalidad de la juventud, de la vejez, etc. Dentro de esta tipología hay naturalmente desarrollos particulares, cultivo consciente de sí mismo, conversiones internas, transformaciones mediante fijaciones éticas de metas, ejercicio, etcétera". ${ }^{39}$

Algunas de las vivencias en las que se expresa mi índole personal peculiar a las que se refiere Husserl en el texto que acabo de citar son claramente de "naturaleza sentimental" (al menos en el esquema interpretativo husserliano) como, por ejemplo,

\footnotetext{
${ }^{36}$ Hua IV, 220/Ideas II, 267

${ }^{37}$ Hua IV, 253-254/Ideas II, 301

${ }^{38}$ Hua IV, 255/Ideas II, 303

${ }^{39}$ Hua IV, 254/Ideas II, 302
} 
el preferir. Sin embargo, en otro texto de Ideas II Husserl deja aún más claro cómo a través de determinados sentimientos puede expresarse quién soy.

Yo, en efecto, no soy solamente el sujeto, el yo que con cierta libertad puede considerar una cosa, mover los ojos viendo, etc. Soy también el sujeto que suele tener agrado en éstas y aquellas cosas, que habitualmente desea esto y aquello, va a comer cuando llega el momento, etc.: sujeto de ciertos sentimientos y costumbres de sentimiento, costumbres de deseo, costumbres de voluntad, tan pronto pasivo, dije, tan pronto activo ${ }^{40}$.

En cualquier caso, se va consolidando un peculiar estilo individual de motivación, una suerte de identidad personal peculiar o "identidad personal en sentido eminente".

Son muchos los temas abiertos en estos análisis y en los que se impone una labor de profundización. En este trabajo hemos intentado mostrar la importancia de una "arqueología"" de la identidad personal que va desde lo que Husserl denomina "fluyente presente viviente" hasta lo que aquí se ha denominado "identidad personal en sentido eminente". En este proceso de conformación y revelación de la identidad personal las vivencias afectivas o sentimientos desempeñan un papel cuyo examen no puede ser pasado por alto.

\author{
Mariano CRESPO \\ Universidad de Navarra \\ ICS, Cultura Emocional e Identidad \\ mjcrespo@unav.es
}

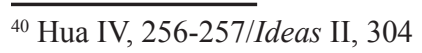

